\title{
ON THE COMPOSITION OF TRANSCENDENTAL ENTIRE AND MEROMORPHIC FUNCTIONS
}

\author{
WALTER BERGWEILER
}

(Communicated by Albert Baernstein II)

\begin{abstract}
It is proved that $f(g)-R$ has infinitely many zeros if $f$ is a transcendental meromorphic, $g$ a transcendental entire, and $R$ a non-constant rational function. The exponent of convergence of the sequence of zeros of $f(g)-R$ is also estimated.
\end{abstract}

\section{INTRODUCTION AND MAIN RESULT}

The main result of this paper is the following theorem.

Theorem. Suppose that $f$ is transcendental meromorphic in the plane, that $g$ is transcendental entire, and that $R$ is a non-constant rational function. Then the equation $f(g(z))=R(z)$ has infinitely many solutions.

K. Katajamäki, L. Kinnunen, and I. Laine [7] proved this result under the hypothesis that $f$ has finite order and $g$ has finite lower order. In fact, they gave a lower bound for the exponent of convergence of the solutions and also dealt with the case that $R$ is transcendental but of smaller growth than $g$. While our method does not seem to be suitable to handle transcendental functions $R$, it does give a bound for the exponent of convergence (compare $\S 3$ ). The results of K. Katajamäki, L. Kinnunen, and I. Laine [7] generalized their results of [6], where they assumed that $f$ is entire, as well as the result of [3], where the above theorem was proved under the additional hypothesis that $f(g)$ has finite order.

The above theorem is known in the case that $R(z)=z$ [2], as well as in the case that $f$ is entire and $R$ is a polynomial [1]. These results confirmed a conjecture of F. Gross [5]. The method used in [2] is based on the observation that $f(g)$ has infinitely many fixpoints if and only if $g(f)$ does. The underlying idea in the present paper is to consider the solutions of $f(g(z))=R(z)$ as fixpoints of $R^{-1}(f(g(z)))$ and to proceed similarly as in [2]. This requires some modifications of the argument, however, because $R^{-1}$ is, in general, not a single-valued function.

Received by the editors November 1, 1993.

1991 Mathematics Subject Classification. Primary 30D05, 30D35.

Key words and phrases. Meromorphic function, entire function, composition, factorization, fixpoint. 
We note that the method of [1] extends to the case that $R$ is rational. It does not seem to extend, however, to the case that $f$ is meromorphic. On the other hand, the case that $f$ has only one pole can be handled by this method with only minor modifications.

\section{PROOF OF THE THEOREM}

We may assume that $R(\infty)=\infty$ and in fact that $R(z) \sim z^{n}$ for some $n \in \mathbb{N}$ as $z \rightarrow \infty$ because otherwise we consider $L(f)$ and $L(R)$ instead of $f$ and $R$ for a suitable linear transformation $L$. In view of the remarks made at the end of the introduction, we may also assume that $f$ has at least two poles $z_{1}$ and $z_{2}$. By $p_{j}$ we denote the order of $z_{j}$.

By [2, Lemma 1] there exist functions $h_{j}$ analytic in a neighborhood of 0 such that $h_{j}(0) \doteq 0$ and $f\left(h_{j}(z)+z_{j}\right)=z^{-p_{j}}$ for $j=1,2$. Similarly as in [2] we define $k_{1}(z)=h_{1}\left(z^{p_{2} n}\right)+z_{1}$ and $k_{2}(z)=h_{2}\left(z^{p_{1} n}\right)+z_{2}$ so that $f\left(k_{1}(z)\right)=f\left(k_{2}(z)\right)=z^{-p_{1} p_{2} n}$.

Next we note that $R(z)=S(z)^{n}$ for some function $S$ which is univalent in a neighborhood of $\infty$ and satisfies $S(z) \sim z$ as $z \rightarrow \infty$. We denote the inverse function of $S$ by $T$. Then $T(z) \sim z$ as $z \rightarrow \infty$ and $R(T(z))=z^{n}$. Finally, following [2], we define $u(z)=g\left(T\left(z^{-p_{1} p_{2}}\right)\right)$ and

$$
v(z)=\frac{u(z)-k_{1}(z)}{u(z)-k_{2}(z)}
$$

Then 0 is an essential singularity of $u$ and hence $v$. Because $k_{1}(0)=z_{1} \neq$ $z_{2}=k_{2}(0)$, we have $v(z) \neq 1$ for sufficiently small $z$. Hence Picard's theorem implies that $v$ takes one of the values 0 and $\infty$ in any punctured neighborhood of 0 . Without loss of generality we may assume that this holds for the value 0 , that is, there exists a sequence $\zeta_{j}$ tending to 0 such that $v\left(\zeta_{j}\right)=0$. It follows that $u\left(\zeta_{j}\right)=k_{1}\left(\zeta_{j}\right)$ and hence that

$$
f\left(g\left(T\left(\zeta_{j}^{-p_{1} p_{2}}\right)\right)\right)=f\left(u\left(\zeta_{j}\right)\right)=f\left(k_{1}\left(\zeta_{j}\right)\right)=\zeta_{j}^{-p_{1} p_{2} n}=R\left(T\left(\zeta_{j}^{-p_{1} p_{2}}\right)\right),
$$

that is, $f\left(g\left(\omega_{j}\right)\right)=R\left(\omega_{j}\right)$ for $\omega_{j}=T\left(\zeta_{j}^{-p_{1} p_{2}}\right)$. The conclusion follows since $\zeta_{j} \rightarrow 0$ implies that $\omega_{j} \rightarrow \infty$.

\section{A QUANTITATIVE VERSION OF THE MAIN RESUlt}

Denote by $\rho(f)$ and $\lambda(f)$ the order and the lower order of $f$ and by $\sigma$ the exponent of convergence of the zeros of $f(g(z))-R(z)$. K. Katajamäki, L. Kinnunen, and I. Laine [7] have shown that $\sigma \geq \lambda(g)$, provided $\lambda(g)<\infty$ and $\rho(f)<\infty$.

We shall show that the slightly stronger inequality $\sigma \geq \rho(g)$ can be obtained without growth restrictions on $f$ or $g$ if we make the assumptions of $\S 2$ :

If $f$ has at least two poles and $R(\infty)=\infty$, then $\sigma \geq \rho(g)$.

To prove this result, we proceed as in $\S 2$. Instead of Picard's theorem, however, we use Nevanlinna theory for functions meromorphic in the neighborhood of an essential singularity (see, e.g., $[4, \S 78 \mathrm{ff}$.$] ). By \rho(u)$ and $\rho(v)$ we denote the orders of $u$ and $v$ at 0 . It is easily seen from the definitions of $u$ and $v$ that $\rho(v)=\rho(u)=p_{1} p_{2} \rho(g)$. By Borel's theorem [4, p. 354], we may assume that the exponent of convergence of $\zeta_{j}$ at 0 is equal to $\rho(v)$ so that $\sum_{j=1}^{\infty}\left|\zeta_{j}\right|^{\mu}$ 
diverges for all $\mu<\rho(v)$. Because $\omega_{j}=T\left(\zeta_{j}^{-p_{1} p_{2}}\right) \sim \zeta_{j}^{-p_{1} p_{2}}$ as $j \rightarrow \infty$, this implies that $\sum_{j=1}^{\infty}\left|\omega_{j}\right|^{-\mu}$ diverges for all $\mu<\rho(g)$, that is, the exponent of convergence of the zeros of $f(g)-R$ is at least $\rho(g)$.

\section{REMARKS}

The estimate $\sigma \geq \rho(g)$ is probably far from being best possible. It seems likely to me that the counting function of the zeros of $f(g)-R$ and the Nevanlinna characteristic of $f(g)$ are always of the same order of magnitude. Possibly the Nevanlinna deficiency $\delta(0, f(g)-R))$ is always equal to 0 if $f, g$, and $R$ are as in the statement of our main theorem. We remark that J. K. Langley [8] proved that if $f$ and $g$ are entire transcendental and if $f(g)$ is of finite order, then $\delta(0, f(g)-R))<1$ for any non-constant rational function $R$. For further results concerning the number of zeros of $f(g)-R$ for entire $f$ we refer to C.-C. Yang and J.-H. Zheng [10].

Finally we mention that the conclusion of our main theorem remains valid if $f$ is a rational function of degree at least 2 (see K. Katajamäki, L. Kinnunen, and I. Laine [7] or G. S. Prokopovich [9]). This follows also from our proof if $f$ has at least two poles.

\section{REFERENCES}

1. W. Bergweiler, Proof of a conjecture of Gross concerning fix-points, Math. Z. 204 (1990), 381-390.

2. - On the existence of fixpoints of composite meromorphic functions, Proc. Amer. Math. Soc. 114 (1992), 879-880.

3. W. Bergweiler and C.-C. Yang, On the value distribution of composite meromorphic functions, Bull. London Math. Soc. 25 (1993), 357-361.

4. A. Dinghas, Vorlesungen über Funktionentheorie, Springer, Berlin, Göttingen, and Heidelberg, 1961.

5. F. Gross, Factorization of meromorphic functions, U.S. Government Printing Office, Washington, DC, 1972.

6. K. Katajamäki, L. Kinnunen, and I. Laine, On the value distribution of composite entire functions, Complex Variables Theory Appl. 20 (1992), 63-69.

7. - On the value distribution of some composite meromorphic functions, Bull. London Math. Soc. 25 (1993).

8. J. K. Langley, On the fixpoints of composite entire functions of finite order, Proc. Roy. Soc. Edinburgh Sect. A 124 (1994), 995-1001.

9. G. S. Prokopovich, Fix-points of meromorphic functions, Ukrainian Math. J. 25 (1973), 198-208; transl. of Ukrain. Mat. Zh. 25 (1973), 248-260.

10. C.-C. Yang and J.-H. Zheng, Further results on fixpoints and zeros of entire functions, Trans. Amer. Math. Soc. 347 (1995), 37-50.

Lehrstuhl II Für Mathematik, RWTH Aachen, D-52056 Aachen, Germany

Current address: Fachbereich Mathematik, Sekr. MA 8-2, TU Berlin, Straße des 17.Juni 136, D-10623 Berlin, Germany

E-mail address: bergweil@math.tu-berlin.de 